\title{
Cuidados dos profissionais de saúde aos idosos com diabetes: um estudo de base populacional no sul do Brasil
}

\author{
Health care for the elderly with diabetes: a population-based study in southern Brazil \\ Atención de los profesionales de la salud a los ancianos con diabetes: un estudio de base poblacional \\ en el sur de Brasil
}

Karla Pereira Machado

ORCID: https://orcid.org/0000-0003-1765-1435 Universidade Federal de Pelotas, Brasil

E-mail: karlamachadok@gmail.com

Michele Rohde Krolow

ORCID: https://orcid.org/0000-0002-7518-7039 Universidade Federal de Pelotas, Brasil

E-mail: michele-mrk@hotmail.com

Marciane Kessler

ORCID: https://orcid.org/0000-0002-4778-8224 Universidade Regional Integrada do Alto Uruguai e das Missões, Brasil E-mail: marciane.kessler@hotmail.com

Adriéli Oliveira Timm

ORCID: https://orcid.org/0000-0002-1174-3569 Universidade Federal de Pelotas, Brasil E-mail: adrielioliveira85@gmail.com

LourieleWachs

ORCID: https://orcid.org/0000-0001-5532-1483 Universidade Federal de Pelotas, Brasil E-mail: louriele@gmail.com

Nicole Pereira Xavier

ORCID: https://orcid.org/0000-0003-2463-3702 Universidade Federal de Pelotas, Brasil E-mail: nicolepxavier@gmail.com

Elaine Thumé

ORCID: https://orcid.org/0000-0002-1169-8884 Universidade Federal de Pelotas, Brasil E-mail: elainethume@gmail.com

\begin{abstract}
Resumo
Objetivo: Avaliar o cuidado prestado pelos profissionais de saúde aos idosos com diagnóstico médico de diabetes mellitus nos serviços de atenção primária no município de Bagé, Rio Grande do Sul. Método: Estudo transversal de base populacional, realizado na zona urbana do município de Bagé, Rio Grande do Sul, com pessoas de 68 anos ou mais pertencentes à coorte SIGa-Bagé em2016/17. O desfecho "cuidado adequado" foi investigado considerando as questões relacionadas aos exames laboratoriais e físicos, realizados no ano anterior à entrevista e do recebimento de orientação sobre a diminuição do consumo de açúcar. A análise descritiva verificou a distribuição proporcional do desfecho e os respectivos intervalos de confiança de 95\% (IC95\%). Resultados: Foram entrevistados 735 idosos. O exame autorreferido mais prevalente foi a glicemia de jejum $(93,2 \%$; IC95\%: 89,1; 97,3) e o exame do pé diabético $(14,5 \%$; IC95\%: 8,7; 20,3) foi o com menor realização. Quanto ao cuidado adequado apenas 1,4\% (IC95\%: -0,1; 3,3) dos idosos tiveram todos os seis indicadores realizados/avaliados. Conclusão: A prevalência de cuidado adequado para diabetes se mostrou aquém do esperado e mostra a fragilidade dos serviços de saúde na atenção. O cuidado deve ser integral, longitudinal e coordenado pela atenção primária. É importante a gestão correta da condição crônica baseada em um plano terapêutico singular e a necessidade de acompanhamento laboratorial e orientações em saúde com foco no indivíduo, família e comunidade.
\end{abstract}

Palavras-chave: Diabetes Mellitus; Assistência integral a saúde; Saúde do idoso; Atenção primária a saúde; Estudos de Coortes.

\footnotetext{
Abstract

Objective: To evaluate the care provided by health professionals to older adults with medical diagnosis of diabetes mellitus in primary health care services in the city of Bagé, Rio Grande do Sul. Method: Cross-sectional population-
} 
Research, Society and Development, v. 11, n. 1, e8811124413, 2022

(CC BY 4.0) | ISSN 2525-3409 | DOI: http://dx.doi.org/10.33448/rsd-v11i1.24413

based study conducted in the urban area of Bagé, Rio Grande do Sul, with people aged 68 years or older belonging to the Siga-Bagé cohort study wave2016/17. The "adequate care" outcome was investigated considering the questions related to laboratory and physical tests performed in the year prior to the interview and the receipt of guidance about the reduction of sugar consumption. The descriptive analysis verified the proportional distribution of the outcome and the respective $95 \%$ confidence intervals $(95 \% \mathrm{CI})$. Results: 735 older adults were interviewed. The most prevalent self-reported test was fasting glucose $(93.2 \%$; 95\%CI: 89.1; 97.3), and diabetic foot examination $(14.5 \%$; 95\%CI: 8.7; 20.3 ) was the one with the lowest prevalence. Regarding adequate care, only $1.4 \%(95 \% \mathrm{CI}:-0.1 ; 3.3)$ of the elderly had all six indicators performed/evaluated. Conclusion: The prevalence of adequate care for diabetes was lower than expected and shows the fragility of health services in care. Healthcare should be comprehensive, longitudinal and coordinated by primary care services. It is important to manage correctly the chronic condition based on a Singular Therapeutic Project and the need for laboratory monitoring, with health guidelines focused on the individual, family and community.

Keywords: Diabetes Mellitus; Comprehensive Health Care; Health of the Elderly; Primary Health Care; Cohort Studies.

\section{Resumen}

Objetivo: Evaluar el cuidado prestado por los profesionales de salud a los ancianos con diagnóstico médico de diabetes mellitus em los servicios de atención primaria em el municipio de Bagé, Rio Grande do Sul. Método: Estudio transversal de base poblacional, realizado em la zona urbana del municipio de Bagé, Rio Grande do Sul, con personas de 68 años o más pertenecientes a la cohorte SIGa-Bagé en 2016/17. El resultado del "cuidado adecuado" fue investigado considerando las cuestiones relacionadas com los exámenes de laboratorio y físicos, realizados el año anterior a la entrevista y La recepción de orientación sobre la disminución del uso de azúcar. El análisis descriptivo verifico la distribución proporcional del resultado y los respectivos intervalos de confianza del 95\% (IC95\%). Resultados: Fueron entrevistados 735 ancianos. La prueba autonotificada más prevalente fue la glucosa en ayunas (93,2\%; IC95\%: 89,1;97,3) y el examen del pie diabético $(14,5 \%$; IC95\%: 8,7; 20,3) fue el con menor prevalencia. En cuanto al cuidado adecuado solo 1,4\% (IC95\%: -0,1; 3,3) de los ancianos tuvieron los seis indicadores realizados/evaluados. Conclusión: La prevalencia de cuidado adecuado para la diabetes se mostró por debajo de lo esperado y muestra la fragilidad de los servicios de salud em la atención. La atención debe ser integral, longitudinal y coordinada por atención primaria. Es importante manejar correctamente la condición crónica en base a un plan terapéutico único y la necesidad de un seguimiento de laboratorio y pautas de salud enfocadas em el individuo, la familia y la comunidad.

Palabras clave: Diabetes Mellitus; Atención Integral de Salud; Salud Del Anciano; Atención Primaria de Salud; Estudios de Cohortes.

\section{Introdução}

O processo de envelhecimento é acompanhado do incremento na ocorrência das condições crônicas (National Institute on Aging, 2018). O Diabetes Mellitus (DM) está entre os principais grupos de Doenças Crônicas Não Transmissíveis (DCNT) que são responsáveis por $71 \%$ das mortes a nível mundial (Organização Mundial da Saúde 2021; Sociedade Brasileira de Geriatria e Gerontologia, 2019)

Estimativas globais referem que 415 milhões de pessoas possuem diagnóstico de DM e, no Brasil, há mais de 13 milhões de pessoas com a doença, o que representa 6,9\% da população (Sociedade Brasileira de Diabetes, 2017).

A incidência de DM aumenta com a idade e está frequentemente associada à mortalidade em idade avançada, destacando que uma proporção considerável é prematura (antes dos 70 anos) (WHO, 2018). Estudos longitudinais nacionais e internacionais evidenciam a diabetes como fator de risco significativo para mortalidade em idosos (De Oliveira et al., 2016; Kessler et al., 2020).

O DM é um distúrbio metabólico cuja principal característica é a hiperglicemia persistente pela deficiência na produção ou na ação da insulina e o acompanhamento dos idosos com esta morbidade requer investimento no autocuidado apoiado ao usuário e familiares. Dentre os fatores de risco mais comuns relacionados com a ocorrência de DM encontra-se a hipertensão arterial sistêmica, a dislipidemia, o excesso de peso, os fatores genéticos e o uso de medicamentos da classe dos glicocorticoides (Sociedade Brasileira de Diabetes, 2017). A elevação das taxas de glicose pode causar complicações microvasculares e macrovasculares como a retinopatia, nefropatia, neuropatia, doenças cardiovasculares e cerebrovasculares, 
Research, Society and Development, v. 11, n. 1, e8811124413, 2022

(CC BY 4.0) | ISSN 2525-3409 | DOI: http://dx.doi.org/10.33448/rsd-v11i1.24413

além disso, agrava outros problemas no sistema musculoesquelético, digestório, cognição e na saúde mental, é associado a alguns tipos de câncer e, em casos graves, pode ocasionar a morte (Sociedade Brasileira de Diabetes, 2017).

A ampliação do acesso aos serviços de saúde, principalmente decorrente da expansão da atenção primária à saúde (APS), possibilitou o diagnóstico e tratamento precoce das condições crônicas, dentre elas, a DM, evitando complicações e mortalidade prematura na população idosa (Kessler et al., 2021). Neste contexto, torna-se imprescindível que o sistema de saúde se organize para atender as necessidades de saúde da população de forma integral e equitativa, com base em medidas de promoção da saúde, prevenção e controle da morbidade (Sociedade Brasileira de Geriatria e Gerontologia 2019; Facchini, Tomasi \& Dilélio, 2018). Além disso, indivíduos com DM devem receber cuidados específicos e realizar acompanhamento através de exames periódicos para avaliar sua condição de saúde e prevenir complicações (Brasil, 2013).

De acordo com o protocolo, os exames de glicemia de jejum e hemoglobina glicada devem ser realizados duas vezes ao ano quando dentro dos valores estabelecidos, caso contrário deve ser realizado a cada três meses. A curva glicêmica e o exame de visão do paciente podem ser solicitados anualmente (Brasil, 2013). Também é preconizado exame físico dos pés, no mínimo, uma vez ao ano com algum profissional de saúde para verificar a presença de sensibilidade e a integridade cutânea, pois sua ausência é sugestiva de lesões e/ou possível amputação. É importante ressaltar que a periodicidade dos exames pode aumentar dependendo da condição de saúde, fatores de riscos e o acompanhamento de cada indivíduo (Brasil, 2013).

O papel do profissional de saúde é essencial na educação em saúde e deve ser contínuo para orientar e acompanhar os cuidados, as mudanças de hábitos e estilo de vida (Brasil, 2013). Neste cenário, destaca-se a atuação dos profissionais da APS, que possuem como competência acompanhar os usuários, estabelecer um cuidado longitudinal, integral e equitativo no território, oferecendo um cuidado de qualidade, baseado nos princípios e atributos da atenção primária (Facchini, Tomasi \& Dilélio, 2018).

No Brasil há poucos estudos de base populacional direcionados para a avaliação do processo de cuidado oferecido pelos serviços de atenção primária à saúde às pessoas com diabetes (Tomasi et al., 2017; Neves et al., 2018). Portanto este estudo tem como objetivo avaliar o cuidado dos profissionais de saúde aos idosos com DM nos serviços de atenção primária no município de Bagé, Rio Grande do Sul.

\section{Metodologia}

Trata-se de um estudo de delineamento transversal de base populacional, em área de abrangência dos serviços de atenção primária à saúde, realizado com a população idosa residente na zona urbana do município de Bagé, Rio Grande do Sul. Este delineamento permite descrever características de uma população com rapidez em sua realização e custo relativamente baixo quando comparado com outros tipos de delineamentos (Gordis L, 2009). Os dados são oriundos do estudo longitudinal intitulado "Situação de Saúde do Idoso Gaúcho de Bagé - RS (SIGa-Bagé)" que teve por objetivo avaliar e acompanhar a situação de saúde dos idosos e a contribuição da Estratégia Saúde da Família (ESF) no atendimento às necessidades em saúde da área urbana de Bagé no período de 2008 a 2016/2017, sendo que para este estudo serão analisados os dados de acompanhamento realizado em 2016/2017.

O município de Bagé situa-se na fronteira do estado do RS com o Uruguai. Em 2016/17 (ano da coleta de dados), residiam no município aproximadamente 122.209 habitantes, dos quais 12,1\% eram idosos, o Índice de Desenvolvimento Humano (IDH) é de 0,740 e a expectativa de vida ao nascer é em média de 75,9 anos (Prefeitura Municipal de Bagé, 2019).

O processo de amostragem foi realizado em 2008, com coleta de dados em múltiplos estágios, respeitando a área de abrangência de cada uma das UBS posteriormente, dividida em microáreas, com identificação numérica de cada quadra, sorteando aleatoriamente o ponto inicial de coleta de dados. Com o intuito de garantir que todos os domicílios tivessem a 
Research, Society and Development, v. 11, n. 1, e8811124413, 2022

(CC BY 4.0) | ISSN 2525-3409 | DOI: http://dx.doi.org/10.33448/rsd-v11i1.24413

mesma probabilidade de compor a amostra e garantir a dispersão desta no território, foi empregado o pulo sistemático de uma a cada cinco residências (Kessler et al., 2020; 2021; Thumé et al., 2021).

O cálculo amostral realizado utilizou como parâmetro uma razão de prevalência de 1,6\%, nível de confiança de $95 \%$, poder estatístico de $80 \%$ e frequência de expostos de 15\%. Foi incluído $10 \%$ para perdas e recusas, $15 \%$ para fatores de confusão e um efeito de delineamento de 1,3. O tamanho da amostra necessário para identificar a prevalência de diabetes foi de 1214 indivíduos. Os cálculos foram realizados pelo programa Epi Info 7.0 (Centers for DiseaseControlandPrevention, Atlanta, Estados Unidos) (Thumé et al., 2021).

Em setembro de 2016 a agosto de 2017, foi realizada uma nova coleta de dados com os mesmos idosos entrevistados em 2008. A população idosa foi constituída por indivíduos com 68 anos ou mais de idade. Os critérios de exclusão no acompanhamento foram estar institucionalizados, ter mudado de município, estar viajando ou não atender ou não encontrado em casa após três tentativas (Thumé et al., 2021).

A coleta de dados foi realizada por entrevistadores previamente capacitados e treinados sendo realizadas no domicílio do próprio idoso. Os entrevistadores tinham uma lista com o nome, telefone, endereço e UBS de referência dos idosos, com o propósito de contatá-los. O questionário utilizado foi estruturado com questões pré-codificadas, padronizado, previamente testadas e aplicado com o uso de aparelho digital Personal Digital Assistent (PDA). O instrumento completo foi composto por 757 perguntas abertas e fechadas, além de realização de medidas antropométricas. Foi realizado um estudo piloto com idosos de uma instituição de longa permanência do município de Bagé, a fim de testar os instrumentos, o manual de instruções, a organização do trabalho de campo e o desempenho dos entrevistadores. A logística previu três tentativas de localização do idoso e aplicação do questionário, caso contrário era considerado perda/recusa, não sendo admitidas substituições (Thumé et al., 2021).

A prevalência de DM foi verificada pela pergunta: "Em algum momento da sua vida algum médico disse que o (a) sr.(a) tem Diabetes (açúcar no sangue), mesmo que controlada? (não/sim)". Se a resposta fosse positiva eram aplicadas as demais questões com suas respectivas categorias de resposta, para caracterizar o desfecho "cuidado adequado": 1) Quando foi a última vez que um médico ou profissional de saúde examinou seus olhos / sua vista (exame de vista) ?; 2) Quando foi a última vez que um médico ou profissional de saúde examinou seus pés para verificar sensibilidade ou presença de feridas ou irritações?Com opções de resposta: nunca fez / há menos de 6 meses / entre 6 meses e menos de 1 ano / entre 1 ano e menos de 2 anos / entre 2 anos e menos de 3 anos / há 3 anos ou mais; 3) O Sr.(a) fez exame de sangue (glicemia de jejum) ?;4) O Sr. (a) fez exame de hemoglobina glicada? Com opções de resposta: não/sim; 5)O Sr.(a) fez exame de curva glicêmica? (Não fiz o exame / Não fiz, mas o exame foi solicitado pelo médico / Sim, fiz o exame) e 6) O Sr. (a) recebeu alguma orientação de um profissional de saúde para ou sobre comer pouco doce ou açúcar? (não/sim). Para as questões 3 a 6 a pergunta se refere ao ano anterior ao mês em que estava sendo realizada a entrevista. Para fins de análise as opções de respostas foram dicotomizadas e considerado "cuidado adequado" quando os idosos tiveram todos os exames realizados e fossem orientados sobre a diminuição do consumo de açúcar até um ano anterior a realização da entrevista.

Foi utilizando o programa Stata 14.0 (StataCorp/College, Estados Unidos da América) para as análises de dados. Foi realizada uma análise descritiva, com o cálculo de proporções e seus respectivos intervalos de confiança de 95\% (IC95\%). Foi verificada a distribuição dos indicadores de atenção à saúde dos idosos com o modelo de atenção da UBS da área de residência (Tradicional/ESF) e com a posse de plano de saúde.

O estudo foi submetido à Plataforma Brasil e avaliado pelo Comitê de Ética em Pesquisa da Faculdade de Medicina da Universidade Federal de Pelotas, aprovado sob parecer 678.664 em 29 de maio de 2014, e conduzido de acordo com os padrões éticos exigidos (Resoluções 466/2012 - 510/2016 - 580/2018), do MS. 


\section{Resultados}

Foram entrevistados 735 idosos em 2016/17. A maioria dos entrevistados era do sexo feminino $(65,4 \%$; n=481) e 82,2\% ( $\mathrm{n}=604)$ da cor branca com idade média de 77,2 (DP $\pm 6,2$ anos). Quanto ao nível de escolaridade 23,2\% (n=169) não havia frequentado escola, 79,7\% $(n=584)$ estavam aposentados, 75,9\% $(n=558)$ dos idosos não moravam sozinhos e 54,4\% $(n=400)$ estavam vinculados ao modelo de atenção à saúde ESF. Quantos aos idosos com DM, a maioria eram mulheres (65,3\%; IC95\% 57,5; 73,1), com idade entre 68 a 79 anos $(69,4 \% ;$ IC95\% 61,9; 76,9) e estavam vinculados ao modelo de atenção ESF (58,5\%; IC95\% 50,4; 49,6)(Tabela 1).

Tabela 1. Descrição da amostra geral e com diabetes melittus de acordo com as características demográficas, socioeconômicas e tipo de modelo de atenção dos idosos da zona urbana de Bagé/RS, 2016/17 (n=735).

\begin{tabular}{|c|c|c|c|}
\hline \multirow{2}{*}{ Variável } & \multirow{2}{*}{$\begin{array}{c}\text { Amostra geral } \\
\mathrm{N}(\%)\end{array}$} & \multicolumn{2}{|c|}{ DiabetesMelittus (n=147 } \\
\hline & & $\%$ & IC 95\% \\
\hline \multicolumn{4}{|l|}{ Sexo } \\
\hline Masculino & $254(34,6)$ & 34,7 & 26,$9 ; 42,5$ \\
\hline Feminino & $481(65,4)$ & 65,3 & 57,$5 ; 73,1$ \\
\hline \multicolumn{4}{|l|}{ Idade } \\
\hline 68 a 79 anos & $505(68,7)$ & 69,4 & 61,$9 ; 76,9$ \\
\hline 80 anos ou mais & $230(31,3)$ & 30,6 & 23,$1 ; 38,2$ \\
\hline \multicolumn{4}{|l|}{ Cor da pele } \\
\hline Branca & $604(82,2)$ & 82,3 & 76,$1 ; 88,6$ \\
\hline Preta, amarela, parda, indígena & $131(17,8)$ & 17,7 & 11,$5 ; 23,9$ \\
\hline \multicolumn{4}{|l|}{ Escolaridade $(\mathrm{n}=729)$} \\
\hline Nenhuma & $169(23,2)$ & 19,9 & 13,$3 ; 26,4$ \\
\hline 1 a 7 anos & $398(54,6)$ & 57,5 & 49,$4 ; 65,6$ \\
\hline 8 anos ou mais & $162(22,2)$ & 22,6 & 15,$7 ; 29,5$ \\
\hline \multicolumn{4}{|c|}{ Classificação socioeconômica*(n=720) } \\
\hline $\mathrm{A} / \mathrm{B}$ & $105(14,6)$ & 14,0 & 8,$2 ; 19,7$ \\
\hline $\mathrm{C}$ & $283(39,3)$ & 46,8 & 38,$6 ; 55,1$ \\
\hline $\mathrm{D} / \mathrm{E}$ & $332(46,1)$ & 39,2 & 31,$1 ; 47,3$ \\
\hline \multicolumn{4}{|l|}{ Aposentado $(\mathrm{n}=733)$} \\
\hline Não & $149(20,3)$ & 27,4 & 20,$1 ; 34,7$ \\
\hline $\operatorname{Sim}$ & $584(79,7)$ & 72,6 & 65,$3 ; 79,9$ \\
\hline \multicolumn{4}{|l|}{ Situação conjugal } \\
\hline Com companheiro/casado & $310(42,4)$ & 46,6 & 38,$4 ; 54,8$ \\
\hline Sem companheiro/solteiro & $105(14,4)$ & 10,3 & 5,$3 ; 15,3$ \\
\hline Viúvo & $316(43,2)$ & 43,1 & 35,$0 ; 51,3$ \\
\hline \multicolumn{4}{|l|}{ Morar sozinho } \\
\hline Não & $558(75,9)$ & 75,5 & 68,$5 ; 82,5$ \\
\hline Sim & $177(24,1)$ & 24,5 & 17,$5 ; 31,5$ \\
\hline
\end{tabular}




\begin{tabular}{lccc}
\hline Modelo de atenção & & & \\
ESF & $400(54,4)$ & 58,5 & 50,$4 ; 49,6$ \\
Tradicional & $335(45,6)$ & 41,5 & 33,$4 ; 49,6$ \\
\hline \multicolumn{1}{c}{ Total } & $\mathbf{7 3 5}(\mathbf{1 0 0 , 0 )}$ & $\mathbf{1 0 0 , 0}$ & - \\
\hline
\end{tabular}

$\mathrm{N}=$ valor absoluto; \% = prevalência; IC95\%= Intervalo de Confiança de 95\%; ESF= Estratégia Saúde da Família.

* Critérios da Associação Brasileira de Empresas de Pesquisa (ABEP). Fonte: Autores.

A prevalência de DM foi de 20,0\% (IC95\%: 17,0; 22,9; n=147). A Figura 1 apresenta a prevalência da realização de cada um dos seis indicadores de atenção ao cuidado à pessoa com DM e a proporção de idosos que realizaram todos os exames. Dos idosos com diagnóstico de DM, observa-se que 93,2\% (IC95\% 89,1; 97,3) realizaram o exame de glicemia de jejum e 29,0\% (IC95\%: 21,5; 36,4) referiram ter tido os olhos examinados no último ano e 14,5\% (IC95\%: 8,7; 20,3) referiram ter seus pés avaliados por um profissional de saúde. E apenas 1,4\% (IC95\%: -0,1; 3,3) dos idosos tiveram todos os seis indicadores realizados/avaliados, ou seja, um cuidado adequado, considerado àquele que realizou todos os exames laboratoriais, físico e recebeu orientação para diminuição do consumo de açúcar (Figura 1).

Figura 1. Prevalência de realização dos indicadores de atenção à saúde dos idosos com Diabetes Mellitus. Bagé, RS, 2016 $(\mathrm{N}=145 *)$.

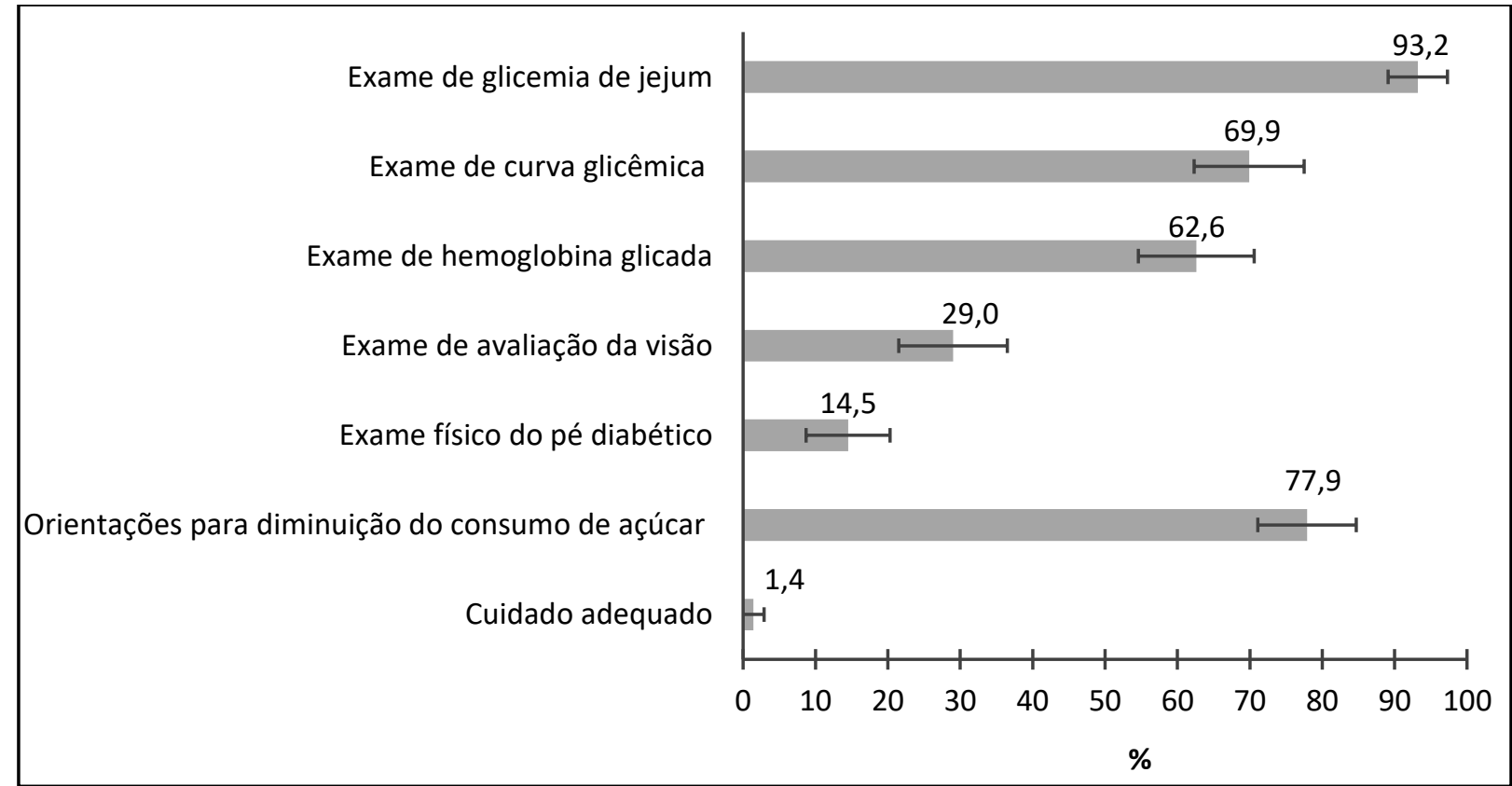

*Perda de uma pessoa na pergunta referente a curva glicêmica e perda e duas pessoas nas perguntas referentes ao exame de olhos, pés e orientação para diminuição de açúcar. Fonte: Autores.

A Figura 2 mostra a prevalência dos indicadores de acordo com a posse de plano de saúde e o modelo de atenção. Foi identificada maior prevalência de realização do exame de hemoglobina glicada, entre os que não tinham plano de saúde, com uma diferença absoluta de cerca de 60 p.p (pontos percentuais) e maior prevalência no recebimento de orientação para diminuir o consumo de açúcar em quem estava vinculado ao modelo de ESF, aproximadamente 68 p.p de diferença com o modelo tradicional. Quanto ao cuidado adequado, não houve diferença em p.p entre os modelos de atenção e entre ter ou não plano de saúde. 
Figura 2. Prevalência (\%) dos indicadores de atenção à saúde dos idosos com Diabetes Mellitus, segundo modelo de atenção e posse de plano de saúde. Bagé, RS, $2016(\mathrm{~N}=145)$.
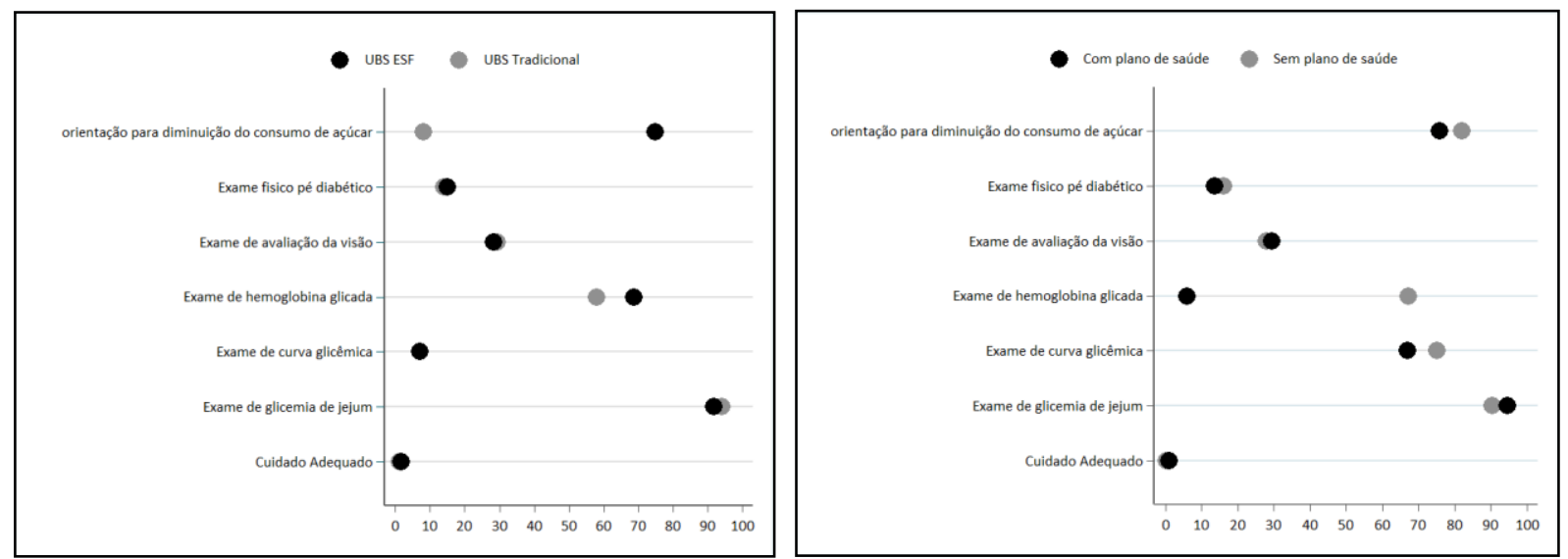

UBS: Unidade Básica de Saúde; ESF: Estratégia Saúde da Família. Fonte: Autores.

\section{Discussão}

O presente estudo teve por objetivo verificar o cuidado prestado pelos profissionais de saúde aos idosos com diagnóstico de DM no município de Bagé (RS) em 2016/2017. Entre os principais achados destaca-se a baixa avaliação da visão e do exame físico dos pés entre os idosos com diabetes. Além disso, aproximadamente apenas um de cada 100 idosos recebeu cuidado adequado, ou seja, todos os exames, avaliações e orientações recomendadas para considerar a qualidade do cuidado.

A prevalência de DM na população idosa acompanhada pelo estudo "SIGa-Bagé" em 2016/2017 teve um incremento de cinco pontos percentuais quando comparada com a prevalência em 2008 (15,1\% e 20,0\%, respectivamente) (Nunes, Thumé \&Facchini, 2015). Vale destacar que a idade média da população entrevistada em 2016 foi maior em relação aos idosos entrevistados em 2008, uma vez que não houve reposição da amostra e, portanto, a população estava mais envelhecida, o que pode influenciar no aumento da prevalência de doenças crônicas (Zhao et al., 2016).

Na Pesquisa Nacional de Saúde (PNS), a prevalência de diabetes foi de 18,2\% na população com 60 anos ou mais (Malta et al. 2019), no Estudo Longitudinal da Saúde dos Idosos Brasileiros (ELSI-Brasil), a prevalência de diabetes ficou em 15,8\% em indivíduos com 50 anos ou mais (Nunes et al. 2018), ambas avaliadas pelo critério de diagnóstico médico de DM autorreferido. Na China, uma pesquisa a nível nacional entrevistou 17.708 idosos e a prevalência foi de 20,1\% na faixa etária de 60 a 69 anos, 21,5\% na faixa etária de 70 a 79 anos e de 26,5\% entre os participantes de 80 anos ou mais de idade, configurando um aumento na prevalência conforme o acréscimo de idade (Zhao et al., 2016).

Além da idade, outros fatores de risco devem ser considerados para a identificação da DM, como os fatores sociais e econômicos, que também são indicadores importantes para determinação de doenças crônicas (Câmara et al., 2019). Cabe ressaltar que o rastreamento de pessoas com DM não diagnosticada deve ser realizado pela atenção primária, visando o tratamento precoce e a prevenção de complicações. Já os indivíduos com diagnóstico de DM devem ter um acompanhamento multiprofissional regular para avaliação dos valores glicêmicos, realização do exame físico e orientações quanto ao estilo de vida e fatores comportamentais (Brasil, 2013).

Neste contexto, a realização de exames laboratoriais é fundamental para monitorar e acompanhar alterações no curso da doença, orientar ajustes nas doses das medicações e atuar precocemente quando há sinais e sintomas sugestivos de 
Research, Society and Development, v. 11, n. 1, e8811124413, 2022

(CC BY 4.0) | ISSN 2525-3409 | DOI: http://dx.doi.org/10.33448/rsd-v11i1.24413

complicações (Brasil, 2013). Quando os níveis glicêmicos se mantêm elevados por longos períodos, há maior susceptibilidade de complicações e desenvolvimento de doenças cardiovasculares, doenças renais, problemas de visão e alterações nos nervos periféricos, amputação parcial ou total de membros, além de aumentar o risco de incapacidade e mortalidade prematura (Brasil, 2013; Sociedade Brasileira de Diabetes, 2017).

O exame da glicemia de jejum neste estudo foi o que apresentou maior prevalência de realização, sendo o mais comum para monitoramento da DM em pessoas saudáveis. Quando o nível glicêmico se encontra instável, outros exames laboratoriais complementares são solicitados para auxiliar no diagnóstico da doença, como o exame da curva glicêmica e da hemoglobina glicada.

Observa-se que aproximadamente um terço dos idosos não realizou os exames de curva glicêmica e hemoglobina glicada no ano anterior a entrevista. O exame da curva glicêmica, também chamado de Teste Oral de Tolerância à Glicose (TOTG), deve ser realizado anualmente e determina a capacidade da pessoa em manter a homeostase da glicose sanguínea após a ingestão oral de uma quantidade de Glicose (Silva, Souza \& Oliveira, 2020). Já o exame da hemoglobina glicada consegue detectar a média da glicemia presente nas células do paciente nos últimos três a quatro meses, sendo assim permite avaliar a sua situação metabólica. Este último é um exame mais completo, traz resultados por períodos mais longos de tempo e a tendência do tratamento é buscar o equilíbrio desse valor (Freitas et al., 2019). Estes exames permitem acompanhar a glicemia daqueles pacientes que têm por hábito se cuidar apenas nos dias que antecedem a coleta, pois não é necessário fazer jejum (Brasil, 2013).

Em um estudo realizado com indivíduos de todas as cinco regiões do Brasil, evidenciou que os exames mais solicitados para a população com diagnóstico de DM eram análise de sangue (glicemia em jejum) (93,1\%), seguido de exame de hemoglobina glicada (72,9\%) (Neves et al. 2018). Outro estudo realizado com diabéticos tipo 2 observou que 62,6\% apresentavam pelo menos uma avaliação de hemoglobina glicada registrada no prontuário e entre estes, 32,0\% apresentavam pelo menos duas avaliações no período de um ano, conforme o recomendado pelo MS (Fernandez et al., 2016). Vale ressaltar que a glicemia de jejum costuma ser o exame de primeira escolha pelos profissionais, pois está na lista de check-up de exames para ser realizado depois dos 40 anos de idade, além de possuir um baixo custo comparado a hemoglobina glicada e a curva glicêmica, o que pode justificar sua maior prevalência em relação aos outros exames laboratoriais.

A avaliação da visão e dos pés foram referidos por menos de 30,0\% dos idosos entrevistados neste estudo. Em um estudo realizado com pacientes diabéticos de uma unidade básica de Campinas (SP) verificou que 62,0\% apresentava alguma complicação relacionada à doença, sendo as mais frequentes, os problemas visuais $(41,9 \%)$ e problemas referentes ao pé diabético (37,1\%) (Maria Bragança et al. 2010). Já em um estudo realizado com 746 indivíduos com DM no ano de 2012, em um município de grande porte do sul do Brasil, observou uma prevalência de $21,1 \%$ de complicações crônicas e entre aqueles com resultados elevados de hemoglobina glicada, 80,6\% apresentou risco para desenvolver úlceras nos pés (Rossaneis et al. 2019).

Estes estudos corroboram com nossos achados e evidenciam a importância e a necessidade destas avaliações para a prevenção de agravos, que podem ser realizadas em qualquer momento na atenção básica ou durante a consulta pelos profissionais de saúde, sendo exames simples e de baixo custo, fundamentais para o rastreio de complicações que devem fazer parte da rotina do exame físico da pessoa com DM.

A avaliação da visão do paciente diabético é essencial para monitorar complicações como o edema macular e a Retinopatia diabética. O edema macular está associado à diminuição da acuidade visual e é tratável. No caso da retinopatia diabética, o excesso de glicose no sangue danifica os vasos sanguíneos dentro da retina causando comprometimento visual. Esta costuma ser assintomática com piora do quadro com o passar dos anos, por isso, quanto antes for detectada mais fácil o 
Research, Society and Development, v. 11, n. 1, e8811124413, 2022

(CC BY 4.0) | ISSN 2525-3409 | DOI: http://dx.doi.org/10.33448/rsd-v11i1.24413

controle. A avaliação da visão do paciente pode ser realizada anualmente, porém acaba variando conforme as necessidades de cada indivíduo (Brasil, 2013).

As úlceras nos pés são uma grave consequência da DM e podem levar a necessidade de amputação de membros inferiores. Essa patologia é causada pela deficiência na circulação sanguínea devido ao descontrole dos valores glicêmicos. Neste sentido, realizar o exame físico dos pés do paciente diabético deve ser frequente e é fundamental a fim de diagnosticar lesões, avaliar perda de sensibilidade, presença de alterações e deformidades que podem sugerir o desenvolvimento de úlceras. A avaliação dos pés deve ocorrer no mínimo uma vez ao ano (Brasil, 2013) e a atenção básica é ponto chave para isso, considerando ser a porta de entrada da maioria dos usuários no SUS, além de ser responsável pelo acompanhamento longitudinal e um cuidado integral (Facchini, Tomasi \& Dilélio, 2018). Além disso, é fundamental orientar o indivíduo ao autocuidado e ensinar aspectos pertinentes para a inspeção diária dos pés (Brasil, 2013).

A avaliação física do indivíduo diabético deve ser minuciosa e requer disponibilidade, escuta atenta, atenção humanizada, vínculo, empatia e respeito. Espera-se que o profissional da saúde explore não somente os problemas do paciente na perspectiva biomédica durante a consulta, mas também voltados ao paciente em seu contexto social e familiar, buscando um cuidado integral que ainda é um desafio na assistência da atenção básica(Comes et al., 2016).

O manejo da DM requer um cuidado contínuo e integral para prevenção de complicações, como a oferta e adesão ao tratamento e exames periódicos e inclui ainda orientações sobre hábitos de vida saudáveis e incentivo ao autocuidado (Venancio, Rosa \& Bersusa, 2016). Neste cenário, apesar da maioria dos idosos terem referido receber orientações para a diminuição do consumo de açúcar, o esperado seria que todos os diabéticos recebessem orientações sobre alimentação. Um estudo com 12.402 pessoas de 100 municípios Brasileiros, somente 28,7\% referiram ter recebido alguma orientação sobre diminuição do açúcar e dentre estes, aproximadamente 83,0\% dos entrevistados percebeu que as orientações para diminuição de açúcar contribuíram para manter uma alimentação saudável (Silva et al., 2013).

Estes resultados sugerem que a proporção de orientações sobre alimentação deve ser menor ainda quando considerada a população em geral, incluindo aqueles que não possuem doenças crônicas. Estudos realizados no Brasil verificaram que indivíduos adultos com doenças crônicas (hipertensão e diabetes) receberam mais orientações sobre alimentação saudável e prática de atividade física do que os indivíduos sem doenças crônicas (Silva et al., 2013; Duro et al., 2015). As práticas de promoção da saúde da atenção básica não devem estar focadas somente nos grupos com problemas crônicos, uma vez que o objetivo principal destas ações é a prevenção da doença. É necessário ainda destacar que estas ações não devem ser impositivas e/ou punitivas, mas desenvolvidas numa relação de vínculo entre profissional e usuário, permitindo a conscientização e o empoderamento para o autocuidado (Kessler et al,. 2018).

Observou-se que um a cada cem idosos com diabetes havia recebido cuidado adequado, ou seja, todos os exames, avaliações e orientações preconizadas no ano anterior a entrevista. Cabe destacar que o exame de visão e do pé diabético foram responsáveis por reduzir a prevalência de cuidado adequado. Um estudo com dados do PNS aponta que, dos 1.685 entrevistados que possuíam diagnóstico prévio de DM, apenas 10,9\% recebeu assistência de todos os oito indicadores avaliados, sendo eles: recomendação para diminuir carboidrato; recomendação para medição de glicose no sangue; recomendação para examinar os pés; pedido de análise de sangue; solicitação de hemoglobina glicada; solicitação de curva glicêmica; olhos examinados no último ano; pés examinados no último ano (Neves et al., 2018), demonstrando uma fragilidade na atenção integral ao indivíduo com DM.

A atenção à saúde do idoso deve ser integral e os cuidados devem ser organizados em rede desde a entrada no sistema. A linha de cuidado deve oferecer ações de educação, promoção da saúde, prevenção de doenças e agravos (Veras, 2016). Fazse necessário colocar em prática as estratégias educacionais voltadas para a DM a fim de garantir a eficácia do autocuidado que 
Research, Society and Development, v. 11, n. 1, e8811124413, 2022

(CC BY 4.0) | ISSN 2525-3409 | DOI: http://dx.doi.org/10.33448/rsd-v11i1.24413

deve ser apoiado. Procura-se promover a conscientização para o desenvolvimento de habilidades, atitudes e comportamentos para o manejo e controle da doença e prevenir complicações (Gomes et al., 2019). A atenção básica é um excelente local para abordar aspectos sociais e emocionais, além de clínicos da doença. A aproximação da equipe de saúde com o indivíduo permite que o plano de tratamento seja o mais individualizado e singular possível integrando a família neste cuidado, fator importante para a adesão ao tratamento e mudança de estilo de vida (Petermann et al., 2015).

Não houve diferença estatística entre os modelos de atenção tradicional e ESF e nem quanto possuir plano de saúde (dados não apresentados), o que sugere que mesmo aqueles pacientes sem condições de obter planos de saúde privados ou que são cobertos por UBS's tradicionais possuem a mesma qualidade de atenção dos que são atendimentos por ESF e possuem planos de saúde. Esperava-se que serviços ligados a ESF tivessem melhor desempenho quanto ao cuidado integral aos pacientes visto que, esse é um programa implantado com o objetivo de melhorar o acesso dos usuários aos serviços. Por outro lado, mostra que serviços gratuitos ligados ao SUS apresentam o mesmo desempenho de planos de saúde. Sendo assim, em ambos os modelos, é necessário que os profissionais de saúde estejam mais atentos em fornecer orientações e em realizar e solicitar os exames, objetivando melhores condições de vida para os idosos diabéticos, a fim de diminuir as consequências causadas pela doença.

Algumas limitações deste estudo podem ser destacadas, como o viés de memória, considerando a possibilidade de que alguns idosos terem dificuldade para lembrar dos exames e avaliações realizadas pelo profissional da saúde; assim como os profissionais de saúde podem ter solicitado os exames ou realizado a avaliação física sem os idosos estarem cientes sobre o tipo de avaliação e sua finalidade. Além da DM autorreferida que pode resultar na redução da estimativa devido casos não diagnosticados previamente, porém acaba se tornando uma escolha pela facilidade, menor custo e rapidez na coleta. A comunicação entre indivíduo e equipe de saúde se faz necessária e deve ser clara, de modo que o indivíduo a ser cuidado possa compreender o cuidado. Como pontos positivos, destacamos o delineamento de base populacional e o rigor metodológico empregado no planejamento, coleta de dados e análises de dados.

\section{Conclusão}

A prevalência de cuidado adequado para diabetes é aquém do esperado e mostra a fragilidade do serviço de saúde na atenção integral. É fundamental o acompanhamento e avaliação periódica dos idosos diabéticos por meio da solicitação de exames laboratoriais, avaliações clínicas, exame físico minucioso e orientações, a fim de evitar outros agravos e complicações, bem como a redução nos anos de vida perdidos por incapacidade e mortalidade prematura.

Estes resultados mostram fragilidades no cuidado ao idoso com diabetes, que deve ser integral, longitudinal, coordenado pela atenção primária e voltada para a pessoa, família e comunidade, considerando as singularidades de cada indivíduo. Destaca-se a necessidade da gestão correta da condição crônica na atenção primária à saúde com base no plano terapêutico singular. Por fim, os resultados podem subsidiar a melhoria da qualidade da assistência ao paciente diabético nos serviços de saúde, especialmente na APS, sendo premente a necessidade de repensar o processo de cuidado, que ainda sofre influência do modelo biomédico.

Sugerimos a realização de estudos que avaliem tanto os relatos dos indivíduos quanto o processo de trabalho das equipes de saúde, a fim de verificar as causas de nem todos os exames necessários para a população idosa com diabetes serem realizados e assim auxiliar na efetivação de programas existentes.

\section{Referências}

Brasil. (2013). Diabetes Mellitus: Caderno de Atenção Básica, No 36. Edited by Ministério da Saúde. 1st ed. Brasília. www.dab.saude.gov.br. 
Research, Society and Development, v. 11, n. 1, e8811124413, 2022

(CC BY 4.0) | ISSN 2525-3409 | DOI: http://dx.doi.org/10.33448/rsd-v11i1.24413

Câmara, S. A. V., Barbosa, T. S., Olivon, V. C., Fernandes, A. L. P, \& Juliana Viana Câmara. (2019). Avaliação do risco para desenvolvimento de diabetes mellitus tipo 2 em estudantes universitários. Revista Ciência Plural 5 (2): 94-110. https://doi.org/10.21680/2446-7286.2019v5n2id18129.

Comes, Y., Trindade, J. de S., Pessoa, V. M., Barreto, I. C. de H. C., Shimizu, H. E., Dewes, D., Arruda, C. A. M., \& Santos, L. M. P. (2016). A implementação do programa mais médicos e a integralidade nas práticas da estratégia saúde da família. Ciencia e Saude Coletiva 21 (9): 2729-38. https://doi.org/10.1590/1413-81232015219.15472016.

Costa, A. F., Flor, L. S., Campos, M. R., Oliveira, A. F. de., Costa, M. de F. dos S., Silva, R. S. da., Lobato, L. C. da P. \& Schramm, J. M. de A. (2017). Carga do diabetes mellitus tipo 2 no Brasil. Cadernos de Saude Publica 33 (2). https://doi.org/10.1590/0102-311x00197915.

Duro, S. M. S., Tomasi, E., Siqueira, F. V., Silveira, D. S., Thumé, E., \& Facchini, L. A. (2015). Adult physical activity counseling by health professionals in Brazil: a national urban population survey. Journal of Physical Activity and Health 12 (8): 1177-83. https://doi.org/10.1123/jpah.2013-0213.

Facchini, L. A., Tomasi, E., \& Dilélio, A. S. (2018). Qualidade da atenção primária à saúde no Brasil: avanços, desafios e perspectivas. Saúde Em Debate 42 (1): 208-23. https://doi.org/10.1590/0103-11042018s114.

Fernandez, N. M., Cazelli, C. \& Teixeira, R. J. (2016). Gerenciamento do controle glicêmico do diabetes mellitus tipo 2 na estratégia de saúde da família. Revista Hospital Universitário Pedro Ernesto (TÍTULO NÃO-CORRENTE) 15 (3): 218-26. https://doi.org/10.12957/rhupe.2016.29447.

Freitas, D. H. F. de, Dias, M. C., Costa, S. H. N., \& Silva, A. M. T. C. (2019). Avaliação do controle glicêmico por meio da a1c, glicemia média estimada e glicemia de jejum em pacientes diabéticos. 51 (1): 70-75. https://doi.org/10.21877/2448-3877.201900798.

Gomes, T. F., Silva, A. P. L., Pereira, B. A., \& Rabelo, N. N. de F. (2019). Desenvolvimento de um programa de educação em diabetes como ferramenta para a promoção da mudança de hábitos de vida: relato de experiência. Atenas Higeia 1 (1): 31-34. https://doi.org/10.2337/dc11-S004.

Gordis L. Epidemiology. 4th ed. Philadelphia: Saunders Elsevier, 2009. p. 247-63.

Kessler, M., Thumé, E., Duro, S. M. S., Tomasi, E., Siqueira, F. C. V., Silveira, D. S., Nunes, B. P. et al. (2018). Ações educativas e de promoção da saúde em Equipes do programa nacional de melhoria do acesso e da qualidade da atenção básica, Rio Grande Do Sul, Brasil. Epidemiologia e Servicos de Saude: Revista Do Sistema Unico de Saude Do Brasil 27 (2): 1-11. https://doi.org/10.5123/S1679-49742018000200019.

Kessler, M., Thumé, E. Marmot, M., Macinko, J., Facchini, L.A., Nedel, F. B., Wachs, L. S. et al. (2021). Family health strategy, primary health care, and social inequalities in mortality among older adults in Bagé, southern Brazil. American Journal of Public Health 111 (5): 927-36. https://doi.org/10.2105/ajph.2020.306146.

Kessler, M., Thumé, E., Scholes, S., Marmot, M., Facchini, L.A., Nunes, B. P., Machado, K. P. et al. (2020). Modifiable risk factors for 9-year mortality in older english and brazilian adults: the ELSA and SIGa-Bagé ageing cohorts. Scientific Reports 10 (1): 1-13. https://doi.org/10.1038/s41598-020-61127-7.

Malta, D. C., Duncan, B. B., Schmidt, M. I., Machado, I. E., Silva, A. G. da., Bernal, R. T. I., Pereira, C. A. et al. (2019). Prevalence of diabetes mellitus as determined by glycated hemoglobin in the brazilian adult population, national health survey. Revista Brasileira de Epidemiologia 22: 1-13. https://doi.org/10.1590/1980-549720190006.supl.2.

Maria Bragança, C., Gomes, I. C., Fonseca, M. R. C. C. da., Colmanetti, M. N. da S., Vieira, M. G. \& Mônica de Fátima Maciel de Souza. (2010). Avaliação das práticas preventivas do pé diabético. J Health Sci Inst 28 (2): 159-63.

National Institute on Aging. (2018). Heart health and aging. 2018. https://www.nia.nih.gov/health/heart-health-and-aging.

Neves, R. G., Duro, S. M. S., Flores, T. R., Wendt, A., Costa, C. dos S., Nunes, B. P., Wehrmeister, F. C. et al. (2018). Social inequalities in care for the elderly with diabetes in Brazil. Revista Panamericana de Salud Pública 42 (88). https://doi.org/10.26633/rpsp.2018.88.

Nunes, B. P., Batista, S. R. R., Andrade, F. B. de., Souza Junior, P. R. B. de., Lima-Costa, M. F. \& Luiz Augusto Facchini. (2018). “Multimorbidade Em Indivíduos Com 50 Anos Ou Mais de Idade: ELSI-Brasil.” Revista de Saude Publica 52 (2): 1-12.

Nunes, B. P., Thumé, E. \& Luiz Augusto Facchini. (2015). Multimorbidity in older adults: magnitude and challenges for the brazilian health system chronic disease epidemiology. BMC Public Health 15 (1): 1-11. https://doi.org/10.1186/s12889-015-2505-8.

Oliveira, C. de., Marmot, M., Demakakos, P., Mambrini, J. V. de M., Peixoto, S. V., \& Maria Fernanda Lima-Costa. (2016). Mortality risk attributable to smoking, hypertension and diabetes among english and brazilian older adults (The ELSA and Bambui Cohort Ageing Studies). European Journal of Public Health 26 (5): 831-35. https://doi.org/10.1093/eurpub/ckv225.

Organização Mundial da Saúde. (2021). Doenças não comunicáveis. 2021. https://www.who.int/news-room/fact-sheets/detail/noncommunicable-diseases.

Petermann, X. B., Machado, I. S., Pimentel, B. N., Miolo, S. B., Martins, L. R., \& Elenir Fedosse. (2015). Epidemiologia e cuidado à diabetes mellitus praticado na atenção primária à saúde: uma revisão narrativa. Saúde (Santa Maria) 41 (1). https://doi.org/10.5902/2236583414905.

Prefeitura Municipal de Bagé. (2019). Plano Municipal de Saúde de Bagé 2018-2021. Bagé. https://www.bage.rs.gov.br/wpcontent/uploads/2019/02/PMS_2018_2021_Oficial_11022019.pdf.

Rossaneis, M. A., Andrade, S. M. de., Gvozd, R., Pissinati, P. de S. C., \& Haddad, M. do C. L. (2019). Factors associated with glycemic control in people with diabetes mellitus. Ciencia e Saude Coletiva 24 (3): 997-1006. https://doi.org/10.1590/1413-81232018243.02022017.

Silva, G. A., Souza, C. L. \& Oliveira, M. V. (2020). Oral glucose tolerance test: unnecessary requests and suitable conditions for the test. Jornal Brasileiro de Patologia e Medicina Laboratorial 56 (January). https://doi.org/10.5935/1676-2444.20200010. 
Research, Society and Development, v. 11, n. 1, e8811124413, 2022

(CC BY 4.0) | ISSN 2525-3409 | DOI: http://dx.doi.org/10.33448/rsd-v11i1.24413

Silva, S. M., Facchini, L. A., Tomasi, E., Piccinii, R., Thumé, E., Silveira, D. S. da., Siqueira, F. V. et al. (2013). Recebimento de orientação sobre consumo de sal, açúcar e gorduras em adultos: um estudo de base nacional. Revista Brasileira de Epidemiologia 16 (4): 995-1004. https://doi.org/10.1590/S1415$790 X 2013000400019$.

Sociedade Brasileira de Diabetes. (2017) . Diretrizes Sociedade Brasileira de Diabetes 2017-2018. Editora Cl. São Paulo. https://www.diabetes.org.br/profissionais/images/2017/diretrizes/diretrizes-sbd-2017-2018.pdf.

Sociedade Brasileira de Geriatria e Gerontologia. (2019) . OMS divulga metas para 2019; desafios impactam a vida de idosos. 2019. https://sbgg.org.br/omsdivulga-metas-para-2019-desafios-impactam-a-vida-de-idosos/.

Thumé, E., Kessler, M., Machado, K. P., Nunes, B. N., Volz, P. M., Wachs, L. W., Soares, M. U. et al. (2021). Cohort study of ageing from Bagé (SIGaBagé), Brazil: profile and methodology. BMC Public Health 21 (1): 1-9. https://doi.org/10.1186/s12889-021-11078-z.

Tomasi, E., Cesar, M. A. D. C., Neves, R. G., Schmidt, P. R. C., Thumé, E., Silveira, D. S. da., Siqueira, F. C. V. et al. (2017). Diabetes care in Brazil program to improve primary care access and quality-PMAQ. Journal of Ambulatory Care Management 40 (2): 12-23. https://doi.org/10.1097/JAC.0000000000000184.

Venancio, S. I., Rosa, T. E. da C. \& Bersusa, A. A. S. (2016). Atenção integral à hipertensão arterial diabetes mellitus: implementação da linha de cuidado em uma região de saúde do estado de São Paulo, Brasil. Physis 26 (1): 113-35. https://doi.org/10.1590/S0103-73312016000100008.

Veras, R. (2016). Care pathway for the elderly: detailing the model" 19 (6): 887-905. https://doi.org/10.1590/1981-22562016019.160205.

Zhao, Y., Crimmins, E. M., Hu, P., Shen, Y., Smith, J. S., Strauss, J., Wang, Y. et al. (2016). Prevalence, diagnosis, and management of diabetes mellitus among older chinese: results from the China health and retirement longitudinal study. International Journal of Public Health 61 (3): $347-56$. https://doi.org/10.1007/s00038-015-0780-x. 